EDITOR'S
CHOICE

General Practice and Primary Care Research Unit, Department of Public Health and Primary Care, Institute of Public Health, University of Cambridge, Cambridge, UK

\section{Correspondence to}

Dr Nahal Mavaddat, General Practice and Primary Care Research Unit, Department of Public Health and Primary Care, Institute of Public Health, University of Cambridge, Forvie Site, Robinson Way, Cambridge CB2 OSR, UK

nm212@medschl.cam.ac.uk

Received 16 June 2010

Accepted 6 August 2010

Published Online First

1 November 2010

\title{
Primary care research and clinical practice: cardiovascular disease
}

\author{
N Mavaddat, J Mant
}

\begin{abstract}
Improvement in survival of patients with cardiovascular diseases and an ageing population mean that management of cardiovascular conditions remains an important challenge for primary care. Traditionally cardiovascular research has been based largely in secondary or tertiary care settings. The majority of care for people with cardiovascular diseases, however, takes place in the community and within primary care. In recent years, progress has been made in conducting cardiovascular research within primary care itself. A number of different methodologies including large prospective cohort studies, randomised controlled trials, and qualitative designs have been used to inform optimal cardiovascular disease management for those in the community. Some of the recent research evidence in primary care in three areas of cardiovascular medicine-atrial fibrillation, heart failure, and cardiovascular risk prediction and management-are discussed in this review. These seek to demonstrate the contribution made by primary care research to the management of cardiovascular diseases.
\end{abstract}

\section{INTRODUCTION}

The last few decades have seen reductions in the age specific incidence, and improvements in outcomes, of cardiovascular diseases. ${ }^{1} 2$ This has been associated with important advances in the evidence base for the prevention and treatment of cardiovascular disease, which have contributed to improved survival and thus increasing prevalence of cardiovascular disease. ${ }^{2-5}$ Ageing of the population accentuates the impact of this increasing prevalence, ${ }^{6}$ and raises the profile of primary care, since this is where the ongoing management of cardiovascular disease and its primary prevention in patients takes place.

\section{THE NEED FOR PRIMARY CARE BASED CARDIOVASCULAR RESEARCH}

One of the problems for primary care in responding to these challenges is that much cardiovascular research has been based in secondary or tertiary care, despite the fact that the majority of care of those with cardiovascular disease takes place in the community and within primary care. Patients with cardiovascular disease in primary care are, however, different to those referred for secondary care management. Furthermore, interventions offered in primary care settings may not be the same as those offered by specialist cardiovascular centres, and the skills and knowledge base of professionals delivering such interventions are not easily comparable (figure 1).
Differences in demographics between study populations in each setting potentially limit the usefulness to primary care of secondary care based trial findings. Patients with heart failure, for example, who present in primary care are about a decade older than those in clinical trials, have more comorbidities, and are more often on multiple medications. ${ }^{78}$ This can alter metabolism, response to medications, and side effects. ${ }^{7} 8$ Similarly, patients requiring thromboprophylaxis in primary care are older and less healthy than those in trials of anticoagulant therapy, since those at greatest risk of complications, particularly the elderly, tend to be excluded from trials due to fears of increased risk of bleeding. 910 Patients from ethnic minorities and women are also often under-represented in secondary care and clinical trials. ${ }^{11}$ For example, women comprise $45 \%$ of those in the community whom guidelines suggest should receive statins to reduce their cardiovascular risk, yet on average comprise only around one sixth of the population in trials of lipid lowering agents, and are underrepresented in trials of secondary prevention after myocardial infarction. ${ }^{11-13}$ Patients in primary care also differ from those in clinical trials in characteristics such as the ability to make frequent repeat visits to the practice for titration of drugs, ${ }^{8}$ and in levels of compliance with medications. ${ }^{14} 15$ These factors may reduce the benefit of therapies considered effective in secondary care trials. In addition, the prevalence of cardiovascular diseases seen in secondary care may not represent the prevalence and hence need for services in primary care.

The skills base between secondary and primary care is also different. For example, the ability of a primary care physician to identify atrial fibrillation or abnormalities on an electrocardiogram (ECG) when heart failure is being considered may be inferior to that of a trained cardiologist in hospital and clinical trials. ${ }^{16} 17$

These differences between primary and secondary care settings contribute to slow translation into primary care practice-for example, the availability of diagnostic tests such as B-type natriuretic peptide (BNP), low uptake of $\beta$ blockade after myocardial infarction and heart failure, poor achievement of targets for risk factor control for blood pressure, cholesterol and glycosylated haemoglobin, and suboptimal use of medications such as antihypertensive and lipid lowering agents in primary care. ${ }^{18-23}$ They also contribute to inequitable use of interventions-for example, underuse of anticoagulation therapy and heart failure treatments in older patients and women, and ethnic and social disparities seen in the management of blood pressure and heart disease 


\section{Differences between Research in Primary and Secondary Care: example of atrial fibrillation}

\section{Different population}

Kalra et al. studied patients in clinical trials of anticoagulants for AF compared to those in the hospital setting and found them to be about $?$ years younger. Yet these patients were still younger than those who make up primary health care teams population of patients with AF ( $48 \%$ were aged over 75 , compared with $58-72 \%$ in studies based in primary care). ${ }^{1-3}$

\section{Different setting}

In the same study by Kalra, INR was measured more frequently in clinical trials than in the hospital setting. Yet in the primary care setting many more patients may be unable or unwilling to travel frequently for INR measurement. 1,4

\section{Different skills}

SAFE study showed that GPs detected 79 out of 99 cases of AF on ECG, but misinterpreted 114 out of 1355 cases of sinus rhythm as $\mathrm{AF}$ (sensitivity $80 \%, 95 \%$ CI $71-87 \%$, specificity $92 \%, 90-93 \%){ }^{5}$

\section{Reference List}

(1) Kalra L, Yu G, Perez I, Lakhani A, Donalds on N. Prospective cohort study to determine if trial efficacy of antico agulation for stroke prevention in atrial fibrillation transl ates into clinical effectiveness. BMJ 2000; 320(7244):1236-1239.

(2) Oswald N. Antic oagulation to prevent stroke in atrial fibrillation. Cohort was younger than many patients with atrial fibrillation in primary care. BM J 2000; 321 (7269): 1156.

(3) Oswald N, Bateman H. Applying research evidence to individuals in primary c are: a study using non-rheumatic atrial fibrillation. Fam Pract 1999; $16(4): 414.419$.

(4) Oliver MD. General practitioners have to decide best ways of allocating their time. BMJ 2000; 321(7269):1156-1157.

(5) Mant J, Fitzmaurice DA, Hobbs FD, Jowett S, Murray ET, Holder R et al. Accuracy of diagnosing atrial fibrillation on electrocardiogram by primary care practitioners and interpretative diagnostic softw are: analys is of data from screening for atrial fibrillation in the elderly (SAFE) trial. BM J 2007; 335(7616):380.

Figure 1 Differences between research in primary and secondary care: example of atrial fibrillation.

management. ${ }^{24-28}$ These examples suggest that much evidence for the management of cardiovascular disease needs to come from primary care itself.

In this review, we will present three areas of primary care cardiovascular research addressing conditions that are important contributors to cardiovascular morbidity and mortality in the community, and in which important and influential contributions have been made by primary care research of various methodologies. These are: (1) atrial fibrillation, (2) heart failure, and (3) cardiovascular risk prediction and management. The benefits to primary care and future directions will also be discussed. For purposes of this review, we focus on studies carried out in the UK primary care system.

\section{ATRIAL FIBRILLATION}

Atrial fibrillation is a major risk factor for stroke and a common cardiovascular problem in the community, which is increasing in prevalence. $^{29} 30$ There is, however, much evidence of its underdetection, and suboptimal and inconsistent management. ${ }^{31-33}$ It is estimated that up to 4500 strokes per year and 3000 deaths in the UK may be preventable through improved services and optimal therapy of atrial fibrillation. ${ }^{34}$ Primary care research has taken a leading role in elucidating issues surrounding prevalence, screening, diagnosis, and management of atrial fibrillation within primary care. This has been predominantly through a number of large randomised controlled trials in primary care populations.

\section{Choice of anticoagulation or antiplatelet therapy}

One such trial, the Birmingham Atrial Fibrillation Treatment of the Aged Study (BAFTA) addressed the relative benefits and harms of warfarin and aspirin in the community management of atrial fibrillation in older people in the community. ${ }^{35}$ Reluctance of primary care doctors to prescribe anticoagulants relates

\section{Methodologies used in primary care cardiovascular} research

- Epidemiological prevalence studies-for example, ECHOES: heart failure in the community.

- Randomised controlled trials-for example, BAFTA: warfarin for atrial fibrillation in the elderly, and complex interventions such as nurse led management of heart failure, patient preference trials.

- Qualitative studies - for example, barriers to patient compliance with medications.

- Nationwide primary care databases - for example, QRISK and ODScore: cardiovascular risk prediction tools developed through use of large shared databases of populations registered with their general practitioner.

- Record linkage studies-for example, studies of medication adherence. 
to both the lack of sufficient data on effectiveness of anticoagulation in older people and pragmatic reasons including concerns over cognitive decline and increased risk of falls. ${ }^{14} 2833$ BAFTA was a randomised controlled trial of patients over 75 with atrial fibrillation who were recruited from a large number of general practices and randomly assigned to warfarin (target international normalised ratio (INR) 2.5) or aspirin (75 mg per day). ${ }^{35}$ After a mean of 2.7 years of follow-up, the results showed a reduced risk of disabling strokes (including intracranial haemorrhages) in elderly patients assigned to warfarin compared to aspirin. ${ }^{35}$ Unlike in secondary care based studies, ${ }^{36}$ it also found no evidence that anticoagulants were more hazardous than aspirin therapy in this age group. ${ }^{35}$ These findings suggest that anticoagulation should be the preferred treatment in the management of atrial fibrillation in older people in primary care. Nevertheless, patient preferences may impact substantially on the clinical decision making process. ${ }^{37}$

\section{Monitoring of warfarin therapy}

Once a decision has been made to anticoagulate patients, however, a further challenge lies in its monitoring through use of the INR. Inadequate control of anticoagulation negates its potential benefits. While INR testing has traditionally taken place in secondary care, increased indications for, and large numbers of patients on, warfarin therapy has resulted in increased involvement of primary care. ${ }^{38}$ Monitoring of patients in primary and secondary care settings may pose different challenges including the frequency with which INR can be measured. A community based randomised controlled trial (RCT) has explored whether oral anticoagulation monitoring can be provided at least as well in primary care as compared with routine hospital management. ${ }^{39}$ Patients were randomised to a practice based anticoagulation clinic which comprised nurse-led near-patient testing and computerised decision making support system (CDSS), or control (hospital clinic) group. The study found after 12 months follow-up that the proportion of time spent in the target INR range was significantly higher for patients in the intervention group, with no significant difference in overall death rates or serious adverse events. This study therefore showed that anticoagulation management can be as safe in primary care settings as in hospital based clinics. ${ }^{39}$

\section{Self management of warfarin therapy}

A further primary care based randomised controlled trial, the Self-Management of Anticoagulation Trial (SMART), ${ }^{40}$ explored whether anticoagulation monitoring could be managed by patients. In this trial, patients on long term oral anticoagulation were randomised to receive training in self management of anticoagulant therapy (self-monitong of INR targets and how to adjust dose) or to routine care (continued attendance at hospital or practice based anticoagulant clinic). The study found that self-management was associated with better INR control in patients whose control had been poor with no increase in the adverse events rate, unlike those in the routine care group whose control showed no improvement. The authors concluded that self management of anticoagulant therapy is effective and safe, and may represent the model of choice for poorly controlled patients. $^{40}$

\section{Screening for atrial fibrillation}

For patients with atrial fibrillation in the community to benefit from treatment with anticoagulation they must first be identified. However, until recently, there has been little evidence as to whether screening for atrial fibrillation in primary care would be a useful policy and, if so, what would be the best method for screening. Studies addressing screening for atrial fibrillation in primary care had been limited, with findings based either on data from single practices or from mixed populations including previously diagnosed atrial fibrillation. ${ }^{41}$ The Screening for Atrial Fibrillation in the Elderly (SAFE) trial was a multicentred cluster randomised controlled trial in patients 65 years and over, with general practices randomly allocated to active screening or usual care. ${ }^{41}$ Patients in active screening practices were further randomised to be invited to attend the practice for systematic screening (at which an ECG was performed) or to opportunistic screening, in which their pulse was taken while attending the practice for another reason (with ECG performed only if the pulse was irregular). The study found the detection rate of new cases of atrial fibrillation per year to be greater in active screening compared to usual care practices and that opportunistic screening was less costly and identified as many cases as systematic screening. ${ }^{42}$ Thus, ECG screening in primary care for atrial fibrillation represents poor value for money in the absence of prior suspicion of atrial fibrillation (i.e. an irregular pulse).

\section{HEART FAILURE}

Heart failure is also becoming increasingly common in an ageing population, ${ }^{43}$ and is a condition in which there has been important primary care based research addressing questions such as the true prevalence of heart failure in the community, as well as issues such as its impact on quality of life and barriers to its effective management in primary care. Various methodologies including prevalence surveys, qualitative studies, and randomised controlled trials have been used for this purpose.

\section{Prevalence and impact of heart failure}

The Echocardiographic Community Heart of England Screening (ECHOES) study conducted within primary care was an important step in establishing the true prevalence of heart failure and left ventricular systolic dysfunction in the community. ${ }^{44}$ The study was one of the world's largest of its kind and consisted of randomly selected patients aged 45 years and older from general practices across a wide geographical spread. ECG and echocardiography (as well as history and examination findings) were used to determine the presence of left ventricular systolic dysfunction (LVSD) on the basis of strict criteria. The study found that approximately $3 \%$ of patients had definite or probable LVSD. ${ }^{44}$ The study also demonstrated that patients with heart failure suffer from a significantly poor quality of life. ${ }^{45}$ Four hundred and thirty-six patients with heart failure were compared with randomly selected controls from the population on quality of life as assessed by the Short Form-36 questionnaire. Those with heart failure had significant impairment on all scales of physical and mental health, in some scales more severe than those with chronic lung disease or arthritis. Qualitative work in the form of interviews with patients with heart failure and with cancer in a community based study has shown that frustration, social isolation, and the stress of balancing and monitoring a complex medication regimen are predominant problems for people with heart failure. This study also contrasted the lack of, and poorly coordinated, health, social, and palliative care services that people with heart failure receive compared to those with cancer. ${ }^{46}$ Findings such as these emphasise the importance of striving to achieve optimal management of patients with heart failure, given the evidence that drug treatment can improve symptoms and survival ${ }^{47} 48$ - especially in light of the fact that heart failure is often misdiagnosed, under-investigated, under-treated and under-dosed with established therapies in primary care. ${ }^{19} 2349-51$ 


\section{Management of heart failure}

Qualitative studies within primary care have provided important insights into why management of heart failure might be suboptimal. Different designs include structured interviews, nominal group techniques, ${ }^{49}$ and focus groups. ${ }^{50} 52$ Difficulties of differential diagnosis and lack of experience in diagnosing and managing heart failure, lack of access to echocardiography, perceived risks of therapy, limited awareness of research evidence and of applicability of research findings to primary care, and local organisational factors were identified as key issues. ${ }^{49} 5052$ Studies of this nature give rise to strategies by which the gap between current practice and guideline based optimal care can be reduced.

One such implementation strategy is to introduce disease management programmes with specialised multidisciplinary follow-up. ${ }^{53}$ However, the applicability of such programmes to primary care has been questioned, since the evidence base has been obtained in highly specialised centres or based on follow-up of patients directly upon discharge from hospitals. ${ }^{53} 55$ A study which brought together elements of nurse-led and multifaceted care for the management of heart failure in the community was a cluster randomised controlled trial including patients with heart failure from primary care practices in which care in the intervention practices was delivered by specialist nurses. ${ }^{53}$ This included assessment, confirmation of diagnoses by investigations, medication management, and liaison between primary and secondary care, with the facility for echocardiography and access to a secondary care cardiology clinic. Patients in the control group received usual care from their primary healthcare team, but also had open access to echocardiography and to the cardiology clinic. The study found that at follow-up significantly more patients with an unconfirmed diagnosis of heart failure had a diagnosis confirmed or excluded in the intervention compared to the control group. ${ }^{53}$ Thus the study contributed to a body of evidence suggesting that nurse-led interventions may improve management of chronic diseases such as heart failure and circumvent some of the barriers to implementation in primary care.

\section{IDENTIFICATION AND MANAGEMENT OF PEOPLE WITH RAISED CARDIOVASCULAR RISK}

Much activity in primary care is directed at identifying people at increased risk of cardiovascular disease. In recent years, risk assessment has moved from identification and treatment of individual risks factors to basing treatment decisions more on an overall assessment of cardiovascular risk for individual patients. ${ }^{56}$ However, there has been concern about the applicability of the tools available to predict cardiovascular risk to patients in the National Health Service, given that the most widely used one was based on a cohort study in the USA - the Framingham study-which did not take into account factors that have been demonstrated to be important in UK settings such as social class and ethnicity. ${ }^{57} 58$

\section{Cardiovascular risk calculation}

One of the important contributions made by primary care to cardiovascular risk assessment has been the design and validation of new cardiovascular disease risk scores, ASSIGN in Scotland and ORISK in England and Wales. ASSIGN was a cardiovascular risk assessment score based on data from a prospective cohort study (the Scottish Heart Health Extended Cohort) of men and women aged 30-74 years, initially free of cardiovascular disease and followed for cardiovascular mortality and morbidity for 10 years. ${ }^{59}$ ASSIGN includes family history and social deprivation (using postcode) and results in better discrimination of risk than the Framingham cardiovascular risk score. By contrast, the ORISK score was developed from analysis of routinely collected data from general practices contributing to the QRESEARCH database consisting of a cohort of 1.28 million patients aged 35-74 years, who were free of existing cardiovascular disease and diabetes. ${ }^{60}$ The latest version of ORISK, QRISK2, in addition to the traditional risk factors such as cholesterol, smoking and blood pressure, also takes account of social deprivation, body mass index, treatment with antihypertensives, family history, ethnicity, rheumatoid arthritis, and atrial fibrillation. ${ }^{61}$ The advantage of the vast size of the database used to construct the score means that conditions of low prevalence in the general population such as rheumatoid arthritis and atrial fibrillation are included as independent predictors. ${ }^{61}$ The advantage of ASSIGN and ORISK2 is that they are derived from a UK population, unlike the Framingham risk score. Further work in risk estimation in primary care has led to the development of a risk calculator for diabetes (ODScore) based upon a further prospective open cohort study using routinely collected data from general practices involving over 2.5 million patients aged 25-79 years free of existing diabetes. ${ }^{62}$

\section{Managing cardiovascular risk}

Once those at risk of cardiovascular disease are identified, steps must be taken to reduce such risks, including encouraging positive behavioural change. However, promoting such change-for example, as in the case of physical activity-among the population is proving a particular challenge for public health and primary care. The ProActive trial evaluated a theory based intervention delivered by trained facilitators to increase physical activity. It was delivered either face-to-face or by phone to sedentary adults with a family history of diabetes and thus at greater risk of diabetes and cardiovascular disease. ${ }^{63}$ It found improvements in physical activity and well-being after 1 year in both people who received the theory based intervention and a brief advice leaflet, and those who received the leaflet only. The intervention was no more effective than the leaflet in increasing physical activity. This finding may be explained by the substantial increase in activity in the control arm, due to trial participation (eg, intensive measurement) and the leaflet. However, it may also be that simple brief interventions are just as effective as more costly ones, such as specific exercise referral. ${ }^{63-65}$ Nevertheless, how to help those at risk adopt positive health behaviours requires further research using interventions with sound theoretical underpinnings for behavioural change and maintenance. ${ }^{63} 66$

\section{Compliance with preventive cardiovascular medications}

Another element of risk control is the use of medications such as antihypertensives, lipid therapy and hypoglycaemic drugs for the purpose of primary cardiovascular disease prevention. However, symptom-free individuals may find it a challenge to comply with taking of medications for the purpose of pure risk reduction. ${ }^{67} \mathrm{~A}$ number of studies, some in primary care, have addressed means of improving patient compliance and possible reasons for non-compliance with cardiovascular medicines. ${ }^{68}$ One qualitative study using interviews of primary care patients on antihypertensives found that patients often have significant reservations and fears about drugs and powerful negative images of medicines. In addition, the attributing of side effects to antihypertensives was widespread. ${ }^{69}$ An exploratory survey among primary care patients with type 2 diabetes using a theory based questionnaire found negative beliefs surrounding the 
taking of hypoglycaemic medications including 'pleasant side effects' held by almost one third of patients. ${ }^{70}$ Another primary care study using focus groups of patients on antihypertensives suggested that patients value greater involvement in decisions surrounding antihypertensive prescribing. ${ }^{71}$ Record linkage studies in primary care have also suggested that monitoring targets such as cholesterol values improves patient adherence to preventive medication. ${ }^{72}$ Such analyses are fundamental first steps in attempting to address the important barrier of non-compliance in improving cardiovascular risk reduction.

\section{IMPLEMENTATION OF FINDINGS OF PRIMARY CARE STUDIES IN CARDIOVASCULAR DISEASE}

In summary, much work has been done in primary care cardiovascular research to generate evidence that is applicable to the primary care setting, to understand better patient and primary care practitioner perspectives, and to help develop innovative new approaches to management of patients with cardiovascular disease.

In the area of atrial fibrillation, much of the findings of studies presented have been brought together in programmes such as 'NHS Stroke Improvement', which coordinates various schemes across the UK. ${ }^{34}$ These include the detection of atrial fibrillation though opportunistic screening (eg, at flu clinics), new models for anticoagulation services in primary and community settings, and local enhanced service schemes for the implementation of decision support tools. ${ }^{73}$ The projects have also included education for patients and primary care workers on palpating pulses and ECG training and interpretation. Evidence is accumulating that such measures are improving the quality of services in the UK and its cost effectiveness. ${ }^{34}$

In the area of heart failure, recognition of the scale of the problem in the community and its present inadequate management, as well as barriers towards such management, identified by primary care research have all informed current practice. For example, steps taken towards improvement in care now include programmes such as the 'Pathways for Heart Failure Care' national priority project and involve improvements such as quicker access to echocardiography, better diagnostic algorithms, efforts to inform prescribing through better diagnosis, uptake of heart failure specialist nurses, home based care, and improvement of palliative care services, as well as attending to psychological and quality of life issues in patients with heart failure. $^{73}$

In the area of cardiovascular risk management, the Department of Health has recently initiated the 'NHS Health Checks', ${ }^{74}$ a programme aimed at patients between $40-74$ years in England, involving a free health check to identify people at risk of cardiovascular disease. The checks will include cholesterol and glucose measurements and recommendation of steps to reduce risk. Innovative research by primary care on issues surrounding consequences of screening, insights into behavioural change, and compliance with preventive medications will contribute to large scale screening programmes as they unfold. Measures to improve non-compliance with medications have been taken up in guidelines on how to improve medicine adherence. $^{75}$

With respect to the determination of an individual's cardiovascular risk, the new primary care based risk calculators which have refined risk identification, are important contributions. In Scotland, the Scottish Intercollegiate Guidelines Network (SIGN) recommends use of the ASSIGN calculator. ${ }^{76}$ In England and Wales, the National Institute for Health and Clinical Excellence (NICE) has not recommended which score to use, but suggests an urgent need to establish which is most acceptable for use in the population. ${ }^{12}$ The ODScore is also a potentially important new contribution to diabetic risk assessment and is available for use both in clinical settings and by the public, and does not require blood glucose measurements. ${ }^{77}$

Finally, in the area of health services, nurse-led and multicomponent complex interventions for cardiovascular risk reduction are being increasingly adopted in primary care. ${ }^{73}$

\section{FUTURE DIRECTIONS IN CARDIOVASCULAR RESEARCH IN PRIMARY CARE}

There is a large and exciting agenda for future research in the field of cardiovascular primary care. An important element of this agenda addresses the need for better early identification and preventive management of those at risk of cardiovascular disease in the community. Further work is needed on how to best identify those at risk in primary care, whether through opportunistic or population screening programmes, and how to best encourage and aid primary care practitioners achieve the aims of reducing cardiovascular risk in the population. One of the exciting areas of ongoing research in reducing the risk of heart disease risk is the identification of newly emerging risk factors, which may help determine those at greatest cardiovascular risk These include, for example, biomarkers such as $\mathrm{C}$ reactive protein (CRP) and lipoproteins and genetic markers, which may predict correlates of poor cardiovascular outcome such as atherosclerotic burden and left ventricular dysfunction. ${ }^{78-82}$ An important research question is whether such emerging new tests would give any additional information to conventional risk factors and whether they are cost effective for adoption within primary care.

In the area of management of cardiovascular risk, an agenda for future primary care cardiovascular research stems from the growing understanding that it is overall cardiovascular risk rather than individual risk factors that may determine the need for intervention. Questions arise as to whether treatments to lower blood pressure, for example, should be given on the basis of overall cardiovascular risk regardless of level of blood pressure. $^{83} 84$ The concept of a polypill to reduce the risk of cardiovascular disease to be given to all individuals over a certain age, regardless of blood pressure reading, is an ongoing area of debate requiring further research and evaluation. ${ }^{85}$

Another research question is how best to monitor risk factors such as blood pressure. The likelihood that observed increases in blood pressure during clinic monitoring reflect true increases, rises with the time between measurements. ${ }^{86}$ This raises questions as to how frequently it is sensible to monitor blood pressure, and the extent to which self monitoring might play a more prominent role. ${ }^{87}$ On the other hand, visit-to-visit variability of systolic blood pressure has been found to be a strong predictor of stroke, independent of mean blood pressure. ${ }^{88}$ The implications of this finding are potentially more frequent measurements of blood pressure, with greater attention to isolated high blood pressure readings when assessing risk and contemplating management. ${ }^{56}$ In the area of cholesterol management, there is also tension as to which is the most effective cost effective strategy for cardiovascular risk reduction between 'fire and forget', where patients are given fixed dose statin therapy without regular monitoring, versus 'treat to target' strategies. ${ }^{12} 7289$ An important agenda for primary care cardiovascular research in the future therefore would be how frequently to monitor risk factor levels such as blood pressure and cholesterol. Linked to the issue of monitoring is the degree to which patients should be involved in their own self care and management, such as blood pressure 


\section{Future directions in primary care cardiovascular research}

- Early identification of cardiovascular disease-for example, early markers of disease.

- Improved diagnosis-for example, clinical decision making rules and near-patient testing.

- Monitoring of disease and risk factors-for example, fewer or more blood pressure readings.

- Self management and monitoring by patients-for example, blood pressure and anticoagulation.

- New treatments-for example, polypill, testing of novel treatments.

monitoring, and whether this will help improve outcomes, an increasingly important part of the management of chronic conditions. 9091

How to improve the diagnosis of cardiovascular disease (eg, through use of near-patient testing) and the care pathways in primary care for those with established conditions such as heart failure is an important question for future research. Improved clinical decision making rules need to be developed and validated. For example, in the diagnosis of heart failure it is important to tease out the relative value of clinical features and investigations such as natriuretic peptide (BNP) testing in order to triage use of echocardiography. ${ }^{92}$

Implementation of research remains high on the agenda for primary care including how to overcome barriers to implementation of new treatments and of evidence based guidelines. Current mechanisms, such as the computerised decision support systems (CDSS), ${ }^{93} 94$ and monetary incentives such as the Quality Outcomes Framework (QOF) pay-for-performance scheme, require continued evaluation. ${ }^{95}$

Understanding reasons for inequalities in cardiovascular healthcare provision is another important area of future work as a prerequisite for developing and testing methods to eliminate them. ${ }^{96}$

Further research is needed into the complex care and rehabilitation of those with chronic cardiovascular conditions such as stroke and heart failure, an area little addressed by secondary care research. The optimal organisation of care within primary care, including who is responsible for what and how patients are monitored, requires further evaluation. This will require greater understanding of how to help patients with multiple

\section{Main messages}

- Cardiovascular disease management takes place mostly within primary care but much evidence is derived from secondary care.

- A broad range of methodologies from large randomised controlled trials to qualitative studies have been used to address the primary care cardiovascular disease agenda.

- Implementation of research findings in primary care has led to improvements in care across a broad range of cardiovascular conditions.

- There is a large and exciting agenda for future research in cardiovascular primary care, including investigation of the role of emerging biomarkers in cardiovascular disease risk identification and new risk factor monitoring strategies. comorbidities, both psychological and physical, an increasingly common problem in the population, ${ }^{97}$ as well as those with complex personal and social circumstances. Patient centred research focusing on patient driven questions is required to address those concerns most important for patients and likely to impact upon their quality of life. ${ }^{98}$ Indeed, who sets the agenda for future cardiovascular research and the balance between patients, health practitioners, the pharmaceutical industry, and not-for-profit national funding agencies will remain a matter for debate. $^{99}$

\section{MULTIPLE CHOICE OUESTIONS (TRUE (T)/FALSE (F); ANSWERS AFTER THE REFERENCES)}

1. When considering applicability of research evidence in clinical trials and secondary care to primary care in cardiovascular disease:

A. Patients with heart failure in trials are around 10 years older than those in primary care

B. Elderly patients with atrial fibrillations have been well represented in trials of warfarin use

C. Women are equally included in clinical trials of cardiovascular drugs

D. General practitioners have been shown to detect atrial fibrillations on ECGs just as well as cardiologists

E. Ability of patients in primary care to make frequent repeat visits for titration of drugs and compliance with medications may reduce the benefit of therapies considered effective in secondary care trials

\section{Research evidence from primary care shows that elderly patients with atrial fibrillation:}

A. Are overly treated with anticoagulants

B. Should not be offered warfarin since they are at increased risk of falls

C. Are at greater risk of bleeding complications with warfarin than aspirin

D. Are best identified through population screening programmes

E. Can have their INR monitored just as well in primary care as in the hospital setting

\section{Research evidence in primary care in the area of heart failure} shows that:

A. About $3 \%$ of patients over the age of 45 have definite or probable LVSD

B. Patients with heart failure have a better quality of life than those with chronic lung disease

C. Patients with heart failure in primary care are on adequate doses of established therapies

D. Nurse-led intervention in the form of nurse-led clinics result in improvements in heart failure management

E. Access to echocardiography and local organisational factors are barriers to effective management of heart failure in primary care

\section{With respect to the investigation and management of} cardiovascular risk in primary care:

A. Risk assessment has moved from identification of individual risk factors to overall cardiovascular risk assessment

B. ASSIGN and ORISK incorporate social class as a factor when assessing risk

C. New studies suggest that visit to visit variability of systolic blood pressure is not as important a predictor of stroke as mean blood pressure 
D. The likelihood that observed increases in BP during clinic monitoring reflect true increases rises with time between measurements

E. The ProActive study showed that in the context of a clinical trial, a more intensive behavioural change programme was no more effective in promoting exercise than a brief intervention leaflet

Acknowledgements NM is supported by an NIHR Clinical Lecturership in Primary Care Research. JM's research is supported by an NIHR Stroke Programme Grant for Stroke Prevention. The views and opinions expressed in this article do not necessarily reflect those of the NIHR.

Competing interests None declared.

Provenance and peer review Commissioned; externally peer reviewed.

\section{REFERENCES}

1. Bennett K, Kabir Z, Unal B, et al. Explaining the recent decrease in coronary heart disease mortality rates in Ireland, 1985-2000. J Epidemiol Community Health 2006:60:322-7

2. Unal B, Critchley JA, Fidan D, et al. Life-years gained from modern cardiological treatments and population risk factor changes in England and Wales, 1981-2000. Am J Public Health 2005:95:103-8.

3. Law MR, Wald NJ, Rudnicka AR. Quantifying effect of statins on low density lipoprotein cholesterol, ischaemic heart disease, and stroke: systematic review and meta-analysis. BMJ 2003;326:1423.

4. Hansson L, Zanchetti A, Carruthers SG, et al. Effects of intensive blood-pressure lowering and low-dose aspirin in patients with hypertension: principal results of the Hypertension Optimal Treatment (HOT) randomised trial. HOT Study Group. Lancet 1998;351:1755-62.

5. Effect of intravenous APSAC on mortality after acute myocardial infarction: preliminary report of a placebo-controlled clinical trial. AIMS Trial Study Group. Lancet 1988:1:545-9

6. Majeed A, Aylin P. The ageing population of the United Kingdom and cardiovascular disease. BMJ 2005;331:1362.

7. King D. Diagnosis and management of heart failure in the elderly. Postgrad Med $\mathrm{J}$ 1996:72:577-80.

8. McDonald K. Current guidelines in the management of chronic heart failure: practical issues in their application to the community population. Eur $\mathrm{J}$ Heart Fail 2005:7:317-21.

9. Oswald N. Anticoagulation to prevent stroke in atrial fibrillation. Cohort was younger than many patients with atrial fibrillation in primary care. $B M J$ 2000;321:1156.

10. Sweeney KG, Gray DP, Steele R, et al. Use of warfarin in non-rheumatic atrial fibrillation: a commentary from general practice. $\mathrm{Br} J$ Gen Pract 1995; 45:153-8

11. Bartlett C, Doyal L, Ebrahim S, et al. The causes and effects of socio-demographic exclusions from clinical trials. Health Technol Assess 2005;9:iii-x, 1

12. National Institute for Clinical Excellence. Cardiovascular risk assessment and the modification of blood lipids for the primary and secondary prevention of cardiovascular disease updated Feb 2010. London: National Institute for Clinical Excellence, 2008

13. Wei L, Ebrahim S, Bartlett C, et al. Statin use in the secondary prevention of coronary heart disease in primary care: cohort study and comparison of inclusion and outcome with patients in randomised trials. BMJ 2005;330:821

14. Gallagher AM, Rietbrock S, Plumb J, et al. Initiation and persistence of warfarin or aspirin in patients with chronic atrial fibrillation in general practice: do the appropriate patients receive stroke prophylaxis? J Thromb Haemost 2008;6:1500-6.

15. Jackevicius CA, Mamdani M, Tu JV. Adherence with statin therapy in elderly patients with and without acute coronary syndromes. JAMA 2002:288:462-7.

16. Jeyaseelan S, Struthers AD, Goudie BM, et al. The Accuracy of ECG Screening by GPS and by Machine Interpretation in Selecting Suspected Heart Failure Patients for Echocardiography. Br J Cardiol 2006:13:216-18.

17. Mant J, Fitzmaurice DA, Hobbs FD, et al. Accuracy of diagnosing atrial fibrillation on electrocardiogram by primary care practitioners and interpretative diagnostic software: analysis of data from screening for atrial fibrillation in the elderly (SAFE) trial. BMJ 2007;335:380.

18. Does bnp testing aid diagnosis of heart failure? Drug Ther Bull 2009;47:110-13.

19. Calvert MJ, Shankar A, McManus RJ, et al. Evaluation of the management of heart failure in primary care. Fam Pract 2009;26:145-53.

20. Carroll K, Majeed A, Firth C, et al. Prevalence and management of coronary heart disease in primary care: population-based cross-sectional study using a disease register. J Public Health Med 2003;25:29-35.

21. Dean SC, Kerry SM, Cappuccio FP, et al. Pilot study of potential barriers to blood pressure control in patients with inadequately controlled hypertension. Fam Pract 2007;24:259-62.
22. Kotseva K, Wood D, De Backer G, et al. EUROASPIRE III: a survey on the lifestyle risk factors and use of cardioprotective drug therapies in coronary patients from 22 European countries. Eur J Cardiovasc Prev Rehabil 2009;16:121-37.

23. Majeed A, Williams J, de Lusignan $\mathrm{S}$, et al. Management of heart failure in primary care after implementation of the National Service Framework for Coronary Heart Disease: a cross-sectional study. Public Health 2005;119:105-11.

24. Ashworth M, Medina J, Morgan M. Effect of social deprivation on blood pressure monitoring and control in England: a survey of data from the quality and outcomes framework. BMJ 2008:337:a2030.

25. Hood S, Taylor S, Roeves A, et al. Are there age and sex differences in the investigation and treatment of heart failure? A population-based study. Br J Gen Pract 2000;50:559-63.

26. Millett C, Gray J, Saxena S, et al. Ethnic disparities in diabetes management and pay-for-performance in the UK: the Wandsworth Prospective Diabetes Study. PLoS Med 2007:4:e191

27. Millett C, Gray J, Bottle A, et al. Ethnic disparities in blood pressure management in patients with hypertension after the introduction of pay for performance. Ann Fam Med 2008;6:490-6

28. Simpson CR, Wilson C, Hannaford PC, et al. Evidence for age and sex differences in the secondary prevention of stroke in Scottish primary care. Stroke 2005; $36: 1771-5$

29. DeWilde S, Carey IM, Emmas C, et al. Trends in the prevalence of diagnosed atrial fibrillation, its treatment with anticoagulation and predictors of such treatment in UK primary care. Heart 2006;92:1064-70.

30. Friberg J, Buch P, Scharling $\mathrm{H}$, et al. Rising rates of hospital admissions for atrial fibrillation. Epidemiology 2003;14:666-72

31. Anderson DR, Gardner MJ, Putnam W, et al. Population-based evaluation of the management of antithrombotic therapy for atrial fibrillation. Can J Cardiol 2005;21:257-66

32. Anderson N, Fuller R, Dudley N. 'Rules of thumb' or reflective practice? Understanding senior physicians' decision-making about anti-thrombotic usage in atrial fibrillation. OJM 2007;100:263-9.

33. Sudlow M, Rodgers H, Kenny RA, et al. Population based study of use of anticoagulants among patients with atrial fibrillation in the community. BMJ 1997; 314:1529-30.

34. Atrial fibrillation, detection and optimal therapy in primary care. NHS Stroke Improvement Programme 2010.

35. Mant J, Hobbs FD, Fletcher $\mathrm{K}$, et al. Warfarin versus aspirin for stroke prevention in an elderly community population with atrial fibrillation (the Birmingham Atrial Fibrillation Treatment of the Aged Study, BAFTA): a randomised controlled trial. Lancet 2007;370:493-503.

36. van Walraven C, Hart $\mathrm{RG}$, Singer $\mathrm{DE}$, et al. Oral anticoagulants vs aspirin in nonvalvular atrial fibrillation: an individual patient meta-analysis. JAMA 2002; 288:2441-8.

37. Man-Son-Hing M, Gage BF, Montgomery AA, et al. Preference-based antithrombotic therapy in atrial fibrillation: implications for clinical decision making. Med Decis Making 2005:25:548-59.

38. Baglin T. Decentralised anticoagulant care. J Clin Pathol 1998:51:89-90.

39. Fitzmaurice DA, Hobbs FD, Murray ET, et al. Oral anticoagulation management in primary care with the use of computerized decision support and near-patient testing: a randomized, controlled trial. Arch Intern Med 2000;160:2343-8.

40. McCahon D, Fitzmaurice DA, Murray ET, et al. SMART: self-management of anticoagulation, a randomised trial [ISRCTN19313375]. BMC Fam Pract 2003:4:11.

41. Fitzmaurice DA, Hobbs FD, Jowett $S$, et al. Screening versus routine practice in detection of atrial fibrillation in patients aged 65 or over: cluster randomised controlled trial. BMJ 2007:335:383

42. Hobbs FD, Fitzmaurice DA, Mant $\mathrm{J}$, et al. A randomised controlled trial and cost-effectiveness study of systematic screening (targeted and total population screening) versus routine practice for the detection of atrial fibrillation in people aged 65 and over. The SAFE study. Health Technol Assess 2005; 9:iii-x, 1.

43. McMurray JJ, Petrie MC, Murdoch DR, et al. Clinical epidemiology of heart failure: public and private health burden. Eur Heart J 1998;19(Suppl):9-16.

44. Davies M, Hobbs F, Davis R, et al. Prevalence of left-ventricular systolic dysfunction and heart failure in the Echocardiographic Heart of England Screening study: a population based study. Lancet 2001;358:439-44.

45. Hobbs FD, Kenkre JE, Roalfe AK, et al. Impact of heart failure and left ventricular systolic dysfunction on quality of life: a cross-sectional study comparing common chronic cardiac and medical disorders and a representative adult population. Eur Heart J 2002; 23:1867-76

46. Murray SA, Boyd $\mathrm{K}$, Kendall $\mathrm{M}$, et al. Dying of lung cancer or cardiac failure: prospective qualitative interview study of patients and their carers in the community. BMJ 2002:325:929

47. Flather MD, Yusuf S, Kober L, et al. Long-term ACE-inhibitor therapy in patients with heart failure or left-ventricular dysfunction: a systematic overview of data from individual patients. ACE-Inhibitor Myocardial Infarction Collaborative Group. Lancet 2000;355:1575-81

48. Shibata MC, Flather MD, Wang D. Systematic review of the impact of beta blockers on mortality and hospital admissions in heart failure. Eur $J$ Heart Fail 2001:3:351-7.

49. Hickling JA, Nazareth I, Rogers $S$. The barriers to effective management of heart failure in general practice. Br J Gen Pract 2001:51:615-18.

50. Horne R, Coombes I, Davies G, et al. Barriers to optimum management of heart failure by general practitioners. $\mathrm{Br} J$ Gen Pract 1999;49:353-7. 
51. Sparrow N, Adlam D, Cowley A, et al. Difficulties of introducing the National Service Framework for heart failure into general practice in the UK. Eur $\mathrm{J}$ Heart Fail 2003; 5:355-61.

52. Fuat A, Hungin AP, Murphy JJ. Barriers to accurate diagnosis and effective management of heart failure in primary care: qualitative study. BMJ 2003;326:196.

53. Khunti K, Stone M, Paul S, et al. Disease management programme for secondary prevention of coronary heart disease and heart failure in primary care: a cluster randomised controlled trial. Heart 2007;93:1398-405.

54. Roccaforte R, Demers C, Baldassarre F, et al. Effectiveness of comprehensive disease management programmes in improving clinical outcomes in heart failure patients. A meta-analysis. Eur J Heart Fail 2005;7:1133-44.

55. Bruggink-Andre de la Porte PW, Lok DJ, van Wijngaarden J, et al. Heart failure programmes in countries with a primary care-based health care system. Are additional trials necessary? Design of the DEAL-HF study. Eur J Heart Fail 2005:7:910-20.

56. Jackson R. Guidelines on preventing cardiovascular disease in clinical practice. BMJ 2000;320:659-61.

57. Anderson KM, Wilson PW, Odell PM, et al. An updated coronary risk profile. A statement for health professionals. Circulation 1991;83:356-62.

58. White C, van Galen F, Chow YH. Trends in social class differences in mortality by cause 1986 to 2000. Health Stat 0 2003:20:25-37.

59. Woodward M, Brindle P, Tunstall-Pedoe H. Adding social deprivation and family history to cardiovascular risk assessment: the ASSIGN score from the Scottish Heart Health Extended Cohort (SHHEC). Heart 2007:93:172-6.

60. Hippisley-Cox J, Coupland C, Vinogradova $Y$, et al. Derivation and validation of QRISK, a new cardiovascular disease risk score for the United Kingdom: prospective open cohort study. BMJ 2007;335:136

61. Hippisley-Cox J, Coupland C, Vinogradova Y, et al. Predicting cardiovascular risk in England and Wales: prospective derivation and validation of QRISK2. BMJ 2008;336:1475-82.

62. Hippisley-Cox J, Coupland C, Robson J, et al. Predicting risk of type 2 diabetes in England and Wales: prospective derivation and validation of ODScore. BMJ 2009;338:b880.

63. Kinmonth AL, Wareham NJ, Hardeman W, et al. Efficacy of a theory-based behavioural intervention to increase physical activity in an at-risk group in primary care (ProActive UK): a randomised trial. Lancet 2008;371:41-8.

64. Harrison RA, Roberts C, Elton PJ. Does primary care referral to an exercise programme increase physical activity one year later? A randomized controlled trial. J Public Health (Oxf) 2005;27:25-32.

65. van Sluijs EM, van Poppel MN, Twisk JW, et al. Effect of a tailored physical activity intervention delivered in general practice settings: results of a randomized controlled trial. Am J Public Health 2005;95:1825-31.

66. Johnston M. Individual and population-based interventions to change lifestyle behaviours: underlying principles and theory. Symposium abstracts. Royal College of Physicians of Edinburgh, 12 March 2009.

67. Gottlieb H. Medication nonadherence: finding solutions to a costly medical problem. Drug Benefit Trends 2000;12:57-62.

68. Schroeder K, Fahey T, Ebrahim S. Interventions for improving adherence to treatment in patients with high blood pressure in ambulatory settings. Cochrane Database Syst Rev 2004;(2):CD004804

69. Benson J, Britten N. What effects do patients feel from their antihypertensive tablets and how do they react to them? Qualitative analysis of interviews with patients. Fam Pract 2006;23:80-7.

70. Farmer A, Kinmonth AL, Sutton S. Measuring beliefs about taking hypoglycaemic medication among people with Type 2 diabetes. Diabet Med 2006;23:265-70.

71. Bane C, Hughes CM, Cupples ME, et al. The journey to concordance for patients with hypertension: a qualitative study in primary care. Pharm World Sci 2007;29:534-40.

72. Wei L, MacDonald TM, Watson AD, et al. Effectiveness of two statin prescribing strategies with respect to adherence and cardiovascular outcomes: observational study. Pharmacoepidemiol Drug Saf 2007;16:385-92.

73. http://www.improvement.nhs.uk/heart. 2010.

74. National Screening Committee. 2nd Report of the UK National Screening Committee. London: Department of Health, 2000

75. National Institute for Health and Clinical Excellence. Medicines adherence: Involving patients in decisions about prescribed medicines and supporting adherence (CG76). London: National Institute for Health and Clinical Excellence, 2009.

76. Scottish Intercollegiate Guidelines Network. Risk estimation and the prevention of cardiovascular disease: a national clinical guideline. Edinburgh: Scottish Intercollegiate Guidelines Network, 2007.
77. http://www.qdscore.org. 2009

78. Melander 0, Newton-Cheh C, Almgren P, et al. Novel and conventional biomarkers for prediction of incident cardiovascular events in the community. JAMA 2009:302:49-57.

79. Newton-Cheh C, Johnson T, Gateva V, et al. Genome-wide association study identifies eight loci associated with blood pressure. Nat Genet 2009.

80. Patel JV, Abraheem A, Creamer J, et al. Apolipoproteins in the discrimination of atherosclerotic burden and cardiac function in patients with stable coronary artery disease. Eur J Heart Fail 2010:12:254-9.

81. Ramachandran S, Vasan RS. Biomarkers of Cardiovascular Disease: Molecular basis and practical considerations. Circulation 2006;113:2335-62.

82. Captoge S, Di Angelantonio E, Lowe G, et al. C-reactive protein concentration and risk of coronary heart disease, stroke, and mortality: an individual participant meta-analysis. Lancet 2010;375:132-40.

83. Law MR, Morris JK, Wald NJ. Use of blood pressure lowering drugs in the prevention of cardiovascular disease: meta-analysis of 147 randomised trials in the context of expectations from prospective epidemiological studies. BMJ 2009;338: b1665.

84. PROGRESS Collaborative Group. Randomised trial of a perindopril-based blood-pressure-lowering regimen among 6,105 individuals with previous stroke or transient ischaemic attack. Lancet 2001;358:1033-41.

85. Wald DS, Wald NJ. The polypill in the primary prevention of cardiovascular disease Fundam Clin Pharmacol 2010:24:29-35.

86. Keenan K, Hayen A, Neal BC, et al. Long term monitoring in patients receiving treatment to lower blood pressure: analysis of data from placebo controlled randomised controlled trial. BMJ 2009:338:b1492.

87. McManus RJ, Mant J. Management of blood pressure in primary care. BM 2009;338:b940.

88. Rothwell PM, Howard SC, Dolan E, et al. Prognostic significance of visit-to-visit variability, maximum systolic blood pressure, and episodic hypertension. Lancet 2010:375:895-905

89. Shepherd J. Mechanism of action of bile acid sequestrants and other lipid-lowering drugs. Cardiology 1989:76(Suppl 1):65-71.

90. Bodenheimer T, Lorig $\mathrm{K}$, Holman $\mathrm{H}$, et al. Patient self-management of chronic disease in primary care. JAMA 2002;288:2469-75.

91. Greenfield S, Kaplan S, Ware JE Jr. Expanding patient involvement in care. Effects on patient outcomes. Ann Intern Med 1985:102:520-8.

92. Mant J, Doust J, Roalfe A, et al. Systematic review and individual patient data meta-analysis of diagnosis of heart failure, with modelling of implications of differen diagnostic strategies in primary care. Health Technol Assess 2009;13:1-207, iii.

93. Hobbs FD, Delaney BC, Carson A, et al. A prospective controlled trial of computerized decision support for lipid management in primary care. Fam Pract 1996;13:133-7.

94. Tierney WM, Overhage JM, Murray MD, et al. Effects of computerized guidelines for managing heart disease in primary care. J Gen Intern Med 2003;18:967-76

95. Campbell SM, Reeves D, Kontopantelis E, et al. Effects of pay for performance on the quality of primary care in England. N Engl J Med 2009;361:368-78.

96. RCGP Health inequalities Standing Group. Addressing health inequalities: A guide for general practitioners. London: Royal College of General Practitioners, 2008.

97. Valderas JM, Starfield B, Roland M. Multimorbidity's many challenges: A research priority in the UK. BMJ 2007:334:1128.

98. Evans H, Thornton H, Chalmers I. Testing Treatments: better research for better healthcare. London: Pinter \& Martin, 2010.

99. Chalmers I, Rounding C, Lock K. Descriptive survey of non-commercial randomised controlled trials in the United Kingdom, 1980-2002. BMJ 2003;327:1017.

\section{ANSWERS}

1. (A) $T ;(B) F ;(C) F ;(D) F ;(E) T$

2. (A) $F$; (B) $F ;(C) F ;(D) F ;(E) ~ T$

3. (A) $\mathrm{T}$; (B) $\mathrm{F}$; (C) $\mathrm{F}$; (D) $\mathrm{T}$; (E)

4. (A) $\mathrm{T}$; (B) $\mathrm{T}$; (C) $\mathrm{F}$; (D) $\mathrm{T}$; (E) $\mathrm{T}$ 